\title{
Friedhelm Hase/Karl-Heinz Ladeur Verfassungsfragen der Curriculum-Revision - Zur juristischen Sanktionierung einer harmonisierenden Pädagogik
}

\author{
I. Vom besonderen Gewalt- zum besonderen Rechtsverbältnis
}

1. Die tradierte Struktur des besonderen Gewaltverhältnisses

Die in Deutschland tradierte juristische Vorstellung faßte die Schule als staatliche Anstalt auf, in der Lehrer und Schüler durch Schulgewalt einerseits und Amtshierarchie andererseits einem »besonderen Gewaltverhältnis « unterworfen waren. War das "allgemeine Gewaltverhältnis « zwischen Staat und Staatsbürger, in dem staatlicher Zwang sich gegenüber der Sphäre rechtlicher Freiheit und Gleichheit gesetzlich legitimieren mußte und mindestens partiell auch gerichtlich kontrollieren lassen mußte, als Verhältnis von Subjekten durch das Recht vermittelt, so bezeichnete das »besondere Gewaltverhältnis « (außer dem Lehrer/Schüler-Verhältnis insbesondere der Status des Beamten allgemein sowie der des Militärs und der Gefängnisinsassen) einen davon separierten, als staatsintern vorgestellten Bereich. Für dieses Verhältnis, dessen Genese einerseits die spezifischen Bedingungen des deutschen Konstitutionalismus reflektierte, der diese Verwaltungsbereiche als »Hausgut der Krone" aus der bürgerlich-parlamentarischen Verfügung ausschloß, in die aber auch die strukturellen Bedingungen »ungleicher «, »unfreier « bürokratischer Disziplin eingingen, konnten die »normalen« Verkehrs- und Vermittlungsformen keine Gültigkeit haben. In diesem Bereich galt prinzipiell nicht Recht, sondern Gehorsam, da der Staat

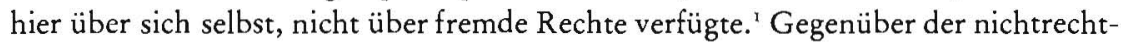
lich vermittelten besonderen Gewalt konnten sich die Gewaltunterworfenen auf Grundrechte nicht berufen. Die tradierte Struktur der Schule implizierte damit die Gleichsetzung der Funktionsweise des gesamten schulischen Prozesses mit derjenigen der allgemeinen staatlichen Verwaltung. Diese Gleichsetzung war möglich, weil durch massive Verdrängung der Besonderheiten des pädagogischen Prozesses der Unterricht sich wie andere staatlich hierarchisch organisierte Veranstaltungen als staatlich durch den (Lehr-)Beamten dargereichte, von den "persönlichen « Interessen des Beamten separierte, nach »unpersönlichen« rationalen Kriterien dargereichte Verwaltungsleistung darstellen ließ, deren Annahme durch den Adressaten, den „unmündigen « Schüler durch Schulpflicht und ein der elterlichen Gewalt nachempfundenes »besonderes Gewaltverhältnis« erzwungen wurde. Die Reduktion der staatlichen Verfügung über Individuen auf die rechtlich nicht determinierte Faktizität der Unterwerfung legitimierte sich in der Vorstellung, daß die Rechtspersönlichkeit im öffentlichen Recht erst durch den Staat in dem je durch die Gesetze bestimmten Umfang "gestiftet « werde ${ }^{2}$.

\footnotetext{
I Zur jurıstıschen Begründung des michtrechtlichen Charakters des besonderen Gewaltverhältnısses wurde auf den zıvilrechtlichen Satz verwıesen, daß eine Person nıcht gegen sıch selbst Rechte und Pflichten haben könne.

2 G. Jellinek: Allgemene Staatslehre, 3. Auflage (7. Neudruck), Bad Homburg v. d. H. 1960, 418 ff.
} 
Das bürokratische, »neutrale«, "sachliche« Bewußtsein, das sich in den Schulen und in den Schülern unter der Form der Identität des Staates mit sich selbst reproduzierte, konnte sich so, als institutionell definierte Idee einer von der Gesellschaft selbst nicht bewußt hervorzubringenden politischen Einheit, mit dem allgemeinen gesellschaftlichen Bewußtsein schlechthin identifizieren ${ }^{3}$.

\section{Funktion und Form der Verrechtlichung des Schulverhältnisses}

Die moderne juristische Auffassung ist derzeit dabei, die besonderen Gewaltverhältnisse zu eliminieren 4 . Auch in der Schule "gelten" nun grundsätzlich die Grundrechte. Auch der schulische Unterricht ist rechtlich gebunden und kann so gerichtlich kontrolliert werden.

Eine quantitative Ausweitung der Rechtsbindungen wurde in der öfentlichen Diskussion und in der Rechtswissenschaft in zunehmendem Maße gerade seit der zweiten Hälfte der sechziger Jahre gefordert - und von der Rechtsprechung auch teilweise realisiert ${ }^{\text {s }}$-, einer Zeit, in der das überkommene Bildungssystem in Frage gestellt wurde. Die vorhandenen Bildungseinrichtungen und -verfahren erwiesen sich angesichts der rasch wachsenden Anforderungen des ökonomischen und politischen Systems zunehmend als antiquiert-dysfunktional und reformbedürftig. $\mathrm{Zu}-$ gleich entstand vor allem im Bereich von Wissenschaft und Ausbildung eine demokratische politische Bewegung, die die anstehende Reform zu beeinflussen und voranzutreiben suchte. Gerade durch die Existenz und Aktivität dieser Bewegung erhielten Bildungspolitik und -reform den für das etablierte politische System prekären Charakter. Dieser Einfluß bedrohte den Konsens zwischen den großen Parteien, der sich auch in der Bildungspolitik auf der Basis relativ starrer Ausbildungsformen und -inhalte herausgebildet hatte. Aus verschiedenen, hier nicht näher zu analysierenden Gründen, war nur eine der beiden großen Parteien, die SPD, willens und in der Lage, ein Konzept für die unabweisbar notwendige Reform zu entwickeln. In dieser Situation entstand eine neue Konstellation: gegenüber dem christdemokratischen Immobilismus mußte es zu partiellen Berührungen und Uberschneidungen zwischen der Politik der SPD und den Forderungen der außerparlamentarischen politischen Bewegung kommen. Die SPD mußte, wollte sie nicht auf Bildungsreformen vollständig verzichten, die Existenz dieser Bewegung in Rechnung stellen und deren Zielsetzung und Forderungen berücksichrigen ${ }^{6}$. Diese Entwicklung provozierte eine Tendenz zur Restriktion der Bildungsreform durch Verrechtlichung, deren Stärke mit der Schwächung der Reforminitiativen selbst zunahm. Für den universitären Bereich sei hier nur auf das Hochschulurteil des

3 Vgl. H. Lefebvre: Das Alltagsleben in der modernen Welt, Frankfurt/M. 1972, 219.

$4 \mathrm{Vgl}$. insbesondere BVerfGE 33, I ff., zum Recht der Strafgefangenen; zum Schulverhältnıs vgl. G. Sturm: Die Schule im Rechtsstaat, in: Recht der Jugend und des Bildungswesens (RdJB) 1974, I ff. m.w.N.

5 Vgl. zur Zulässıgkeıt des "Schulgebets« nur BVerwG, ın: Die Offentliche Verwaltung (DOV) 1974, 278; E.W. Böckenförde: Vorläufige Bilanz im Streıt um das Schulgebet, DOV r 974, 253 ff. m.w.N.; OVG Berlin, in: Juristenzeitung (JZ) 1973 , 55 i ff. m. Anmerkung von H. U. Evers, a. a. O., 555 f.; OVG Hamburg, in: Sammlung schul- und prüfungsrechtlicher Entscheidungen (SPE) I A VI, i i e (beide Entscheidungen zur Verfassungsmäßigkett des Sexualkundeunterrıchts)

6 Die Fähigkeit der SPD zur „Abnahme« progressıver Reformmodelle schennt uns gerade in dem etatıstıschen Reformısmus dieser Partet begründet zu seın: Die von den gesellschaftlichen Produktıonsprozessen organısatorısch abgetrennten Bildungsinstıtutionen reproduzieren die Bedingungen der Abtrennbarkeit von Bildungsınhalten, die so ebenso wie die Institutionen selbst als Gegenstand staatlicher Reformpolitik erscheınen, die anscheınend gesellschaftlich artikulierte Bedürfnisse verarbeiten kann, ohne dadurch gezwungen zu sein, die gesellschaftlichen Strukturen selbst, unter denen diese Bedürfnısse produziert werden, in Frage stellen zu müssen. 
Bundesverfassungsgerichts sowie auf die fast unübersehbare Zahl der Entscheidungen zum sogenannten Politischen Mandat der Studentenschaften verwiesen. Diese Verrechtlichung des Bildungswesens dient generell dazu, die Entwicklungsbedingungen außerparlamentarischer politischer Bewegungen einzuschränken und dadurch insbesondere zu verhindern, daß diese Einfluß auf die sozialdemokratische Bildungspolitik gewinnen und dadurch den Konsens zwischen den "großen « Parteien gefährden.

Im Schulwesen wird insbesondere die formale Eliminierung des »besonderen $\mathrm{Ge}$ waltverhältnisses « zum Vehikel der Verrechtlichung der Bildungsreform. Unter der Form der bloß einfachen Negation des nicht-rechtlichen Charakters des besonderen Gewaltverhältnisses reproduziert sich die Grundstruktur der Schule: Die besonderen Bedingungen des in der Schule organisierten gesellschaftlichen Bildungsprozesses blieben dadurch verdrängt, daß umstandslos nicht nur Entscheidungen (Nichtversetzung, Schulstrafen etc.) über Individualpositionen der Schüler, sondern weitgehend auch die Vermittlung der Bildungsinhalte in das Schema der verbindlichen Verwaltungsrechtsentscheidung transportiert wurden. Diese antithetische Fixierung auf die besondere Gewalt des besonderen Gewaltverhältnisses, in dem der Staat über sich selbst als Subjekt verfügte, mußte dieses umstandslos in das »besondere Rechtsverhältnis« überführen, in dem der Staat aufgrund besonderen Rechts über fremde Subjekte verfügt. Dadurch konnte auch die staatliche Bestimmung über die Bildungsinhalte sich - in scheinbarer Angleichung an das allgemeine Gewalt- (= Rechts-)Verhältnis - wie ein je im Einzelfall rechtlich zu legitimierender Eingriff in Freiheitsrechte darstellen. Der Unterschied zum besonderen Gewaltverhältnis besteht nur darin, daß die rechtliche Persönlichkeit des Kindes nicht mehr durch Integration in die Persönlichkeit des Staates generell aufgehoben bleibt, sondern ihr Freiheitsraum je im Einzelfall von dem der Rechtspersönlichkeit des Staates rechtlich abgegrenzt wird und damit formal eine gerichtliche Kontrolle ermöglicht wird, ohne daß dies materiell eine reale Freisetzung von Handlungsmöglichkeiten des Schülers und von innovierenden Entwicklungsmöglichkeiten des pädagogischen Prozesses insgesamt impliziert.

Im folgenden soll zunächst die Rechtsstellung des Schülers nach der Konzeption der "modernen " Grundrechtsdogmatik dargestellt werden und im Anschluß daran sollen die Bedingungen und Formen der rechtlichen Restriktion der didaktischen Reform näher untersucht werden.

\section{Die »Normalität* als Drehpunkt der Verrechtlichung der Schule}

I. Der »normale« Grundrechtsgebrauch

Die Funktionsweise des oben beschriebenen konstruktiven Ansatzes manifestiert sich exemplarisch in der Vorstellung, das Recht des Kindes auf freie Entfaltung seiner Persönlichkeit (Art. 2 Abs. I GG) werde durch Lehrpläne etc. eingeschränkt’. Diese Konzeption macht nicht nur eine der Verrechtlichung stets immanente Tendenz deutlich, Prozesse (hier der Persönlichkeitsentwicklung) in Verhältnisse zwischen Personen, die durch rechtlich bestimmte "Schranken « begrenzt werden, darzustellen, sie zeigt auch, daß die Entwicklungsmöglichkeiten des Kindes unter dieser Form nunmehr als rechtliche Defizite begriffen werden, die ihr Pendant in entsprechend erweiterten Rechten des Staates finden. Diese rechtliche Grundstruk-

7 Vgl. nur VGH Baden-Württemberg, ESVGH $I$ I, , ff. 
tur bestimmt auch die Dogmatik insbesondere der politischen Grundrechte: Daß die Grundrechte im besonderen Rechtsverhältnis grundsätzlich »gelten«, wird kaum mehr bestritten, auch juridische Konstruktionen von speziellen, dem Zivilrecht nachempfundenen Grundrechtsschranken für Minderjährige ("Grundrechtsmündigkeit«) haben vor der "Würde der Verfassung « kaum mehr Bestand. Wegen der "wertsetzenden Bedeutung « der Grundrechte für die freiheitliche demokratische Ordnung müssen sich alle, auch die gesetzlich vorgesehenen Grundrechtsschranken vor dem »Wertsystem « der Verfassung legitimieren. Die Kollision von prinzipiell gleichrangigen "Werten", die durchaus nicht nur durch die Grundrechte, sondern auch durch die organisatorischen Normen des Grundgesetzes begründet werden ${ }^{8}$, führt nun statt zu größerem Schutz der Grundrechte zur normativen Entfaltung einer insbesondere vom Bundesverfassungsgericht zur Legitimität eıner Überverfassung stilisierten »Normalität«, an deren Maßstab die Lösung der "Güterkollision « durch "Abwägung " vorzunehmen ist. Die "Güterabwägung " setzt sich selbst als Referenzebene neben die positivierte Verfassung, die in der "Vagheit« ihrer Rechtsbegriffe der Entwicklung des polittschen Prozesses zuviel Raum zu geben scheint. Den durch die Auflösung positivierten Verfassungsrechts in konfligierende Rechtsgüter geschaffenen »Nullpunkt « hält das Gericht besetzt. Durch die bloße institutionelle Praxis des "Urteilens" wird das, was es selbst als normal beurteilt, in Normativität transformiert, indem das "neutrale "Gericht zwischen den Gütern als "Parteien«, die um »ihr « Recht streiten, einen Kompromiß findet, der für beide gleichermaßen verbindlich ist, eben: weil es »Recht spricht«. (Die Güterabwägung ist eine typisch juristische Tautologie, die sich nur hinter einem autoritären Argument verschanzen kann: "So antworten Eltern, die mit ihrem Latein am Ende sind, ihrem fragenden Kind: , Das ist so, weil es so ist! « ${ }^{8 a}$ ) Für das Schulverhältnis stellt sich dies so dar, daß der Schüler seine Freiheit - und auf eine dadurch vermittelte Weise auch der Lehrer - einer Erziehungsaufgabe des Staates konfrontiert sieht, deren "Normalität« auf die Ebene der Verfassung transportiert worden ist und dort unter der Form einer bloßen Kompetenzverlagerung die Wiedergeburt des alten besonderen Gewaltverhältnisses bewirkt: die früher durch die besondere Gewalt der Verwaltung definierte Erziehungsaufgabe wird nun durch das von der Rechtsprechung entwickelte besondere Recht rechtsverbindlich definiert. Die besondere Gewalt über unmündige Schüler wird in den Darstellungsbedingungen der gerichtlichen Praxis transformiert in das Recht des Staates, zur »Persönlichkeit « zu erziehen, denn Grundrechtsausübung setzt »Persönlichkeiten « voraus, zu denen die Schüler »durch Erziehung und Unterrichtung in den Schulen erst allmählich herangebildet werden können «?. Schloß früher die von der Schulverwaltung fixierte Schuldisziplin die Berufung auf Grundrechte aus, so wird durch die moderne Grundrechtsdogmatik die in letzter Instanz vom Gericht definierte Schuldisziplin zum Maßstab sowohl der als "normal« anzusehenden Ausübung von Grundrechten wie der Ausübung des staatlichen Erziehungsrechts. Diese "moderne « Dogmatik ermöglicht einerseits eine Erweiterung der gerichtlichen Kontrolle pädagogischer »Urteile (Nichtversetzung etc.) jedenfalls, soweit der pädagogische "Beurteilungsspielraum" überschritten wird, und andererseits - und dies soll hier näher untersucht werden - die Verrechtlichung einer tradierten Form von »Pädagogik als Güterabwägung«, die durch

\footnotetext{
8 Vgl. z. B. zum Verfassungsrang der Bundeswehr BVerfGE 28, $243 \mathrm{ff} . ;$ kriusch dazu O. E. Kempen: Staatsraison über Verfassungsraison?, JZ 1971, 4 S2 ff.

8a R. Barthes: Mythen des Alltags, 2. Aufl., Frankfurt/M. 1970, S. 143.

9 So exemplarısch A. Hildebrandt: Das Schulwesen als Bestandieil der rechtsstaatlichen Hohertsverwaltung, Diss. Marburg 1971, 139.
} 
gerichtliche Güterabwägung verbindlich institutionalisiert wird und dadurch die Entwicklungsmöglichkeiten einer alternativen Pädagogik beschränkt.

\section{Harmonisierende und realistusche Pädagogik}

Die traditionelle Zentrierung der Bildungsinhalte im "Lehrplan « ist nicht, "wie es manchmal scheint, ein Streit um die besten Methoden im Unterricht oder um die Auswahl und Verteilung eines gegebenen Stoffes : $^{\circ}$. Der Lehrplan ist in der Theorie Wentgers, eines Vertreters der traditionellen harmonisierenden Pädagogik, der immerhin Widersprüche überhaupt zur Kenntnis nimmt, begrifflicher Niederschlag der »inneren Form « des Staates, der selbst als "Träger des Lehrplans « die »Einheit des geistigen Lebens im "Kampf geistiger Mächte herstellt ${ }^{\prime \prime}$. Dieser Ansatz ist nicht etwa nur eine idealistische Fiktion, sondern er verallgemeinert in einer theoretischen Form die reale Fiktion der »zweckfreien Bildung ", deren praktische Form die "verwaltete Schule « ist ${ }^{12}$. Diese Theorie der Bildung tritt mit dem Anspruch auf, "vorgegebene inhaltliche Forderungen an die Erziehung zu überprüfen und in bildungsgerechte Formen zu übertragen, in denen sie ihren bedingungslosen Durchsetzungswillen verlieren « $^{13}$. Der Bildungsbegriff fungiert als »Katharsis der gesellschaftlichen Interessen, die sich in der Staatsperson als Instanz des "Gemeinwohls " herstellt und sich durch die (Schul-)Bildung in der gebildeten Persönlichkeit reproduzieren soll.

Der "Besinnungsaufsatz" ist die schulische Praxisform dieser sprachlichen Bildung des Subjekts par excellence: Sie inszeniert einen "Dialog « zwischen verschiedenen kontroversen Positionen (Sollen unsere Eisenbahner Uniform tragen? Kino oder Theater? Was ist Freiheit? usw. usw.), die, anscheinend miteinander unvereinbar, ihre Kohärenz nicht im realen gesellschaftlichen Funktionszusammenhang - dieser Zusammenhang wird in dieser Form gerade systematisch verdrängt - sondern in der durch rhetorische Formen der "Überhöhung « und "Vertiefung « begrenzten Grenzüberschreitung findet, die alle Unterschiede in der Einheit des Subjekts, das sich selbst als "denjenigen, der alle Fäden der Argumentation in der Hand hält «'4, inszeniert. Die Variabilität der mehreren wvertretbaren « Lösungen, die sich dem Individuum wie zur Selektion und zur Interpretation anbieten ${ }^{15}$, lassen die schulischen Formen der "Abwägung ", die besondere Form, unter der die eher privilegierten "Oberschüler« die Aneignung der herrschenden, »normalen « Ideologien vollziehen, als »Freiheit « erleben. Die »Volksschüler« üben demgegenüber in der traditionellen Schule sprachliche Techniken in einer Form ein, die vorwiegend durch ihre konkreten Bezüge zu einem bestimmten Ausschnitt von »Wirklichkeit « sich wie eine deskriptive Abbildungstechnik darstellt, deren Beherrschung sich dem Lernenden mit "technischer" oder sonst "natürlicher " Autorität als äußerlich aufzwingt ${ }^{16}$, während der "Oberschüler « seine Lösungen selbst entdeckt. Das

to E. Wenıger: Didaktik als Bildungslehre, Teil I - Theorıe der Bildungsınhalte und des Lehrplans, 4. Aufl., Weinheim 1962, 22 (zitıert nach: F Hulsken: Zur Krıtik bürgerlicher Didaktik und Bildungsökonomı, München 1973, 47).

II E. Weniger, a. a. O., 33, 36 .

I2 E. Wentger, a. a. O., 62; vgl. dazu F. Hutsken, a. a. O., $47 \mathrm{ff}$.

13 H. Blankertz: Theorıen und Modell der Didaktik, München 1969, r 21 (zituert nach $F$ Husken, a. a. O., SI).

${ }_{4}$ Ch. Baudelot/R. Establet: L'école capitaliste en France, Parıs 1972, 192

is Vgl. zur literarischen "Interpretation " E. Balibar/P Macherey: Thesen zum materialistischen Verfahren, In: Alternative $98,193 \mathrm{ff}$.

i6 Ch. Baudelot/R. Establet, a. a. O., I52. 
Verhältnis der Trennung der sprachlichen Praxisform der eher »literarisch « orientierten traditionellen "Oberstufe " und der eher "technisch-deskriptiv « orientierten "Volksschule « artikuliert sich als abstufende Differenzierung und Selektion innerhalb der Einheit einer formal-kognitiven, von den gesellschaftlichen Produktionsbeziehungen distanzierten "Qualifikation «"

Diese einheitliche, von der Produktionspraxis abgelöste, vor allem sprachliche Qualifikations-"praxis" setzt ihr Maß selbst in Prüfungen, für alle gleiche, nur nach der "Leistung " differenzierenden Noten etc. und bringt so die Schüler zur "Anerkennung des legitimen Wissens und Könnens ${ }^{18}{ }^{18}$ und damit zugleich zur Anerkennung der Trennung der Schüler als Ausdruck unterschiedlicher Grade von subjektiver Qualifikation.

"Bildungsreform als Revision des Curriculum" (Saul B. Robinsohn), wie sie vor allem mit den Entwürfen der hessischen Rahmenrichtlinien ${ }^{19}$ intendiert war, versuchte, nicht nur neue Inhalte in neuen Lehr- und Lernplänen zu formulieren, sondern die Grundstruktur des Schulverhältnisses in dem Sinne zu verändern, daß den Schülern - vermittelt durch die Lehrer - nicht mehr ein "Lehrstoff « als Staatsleistung verabreicht werden soll, sondern durch die Formulierung von Lernzielen, die erst die Bildungsinhalte als ihre Funktion determinieren, die entsprechend variabel sind und sich zunächst orientieren an gesellschaftlichen ¿Situatio$n e n *$, denen die Schüler sich konfrontiert sehen, und den dadurch den Schülern abverlangten sozialen Verhaltensweisen ${ }^{20}$. Der Lernprozeß soll auf Handlungen orientiert werden, an deren Bedingungen gemessen werden kann, »wozu Gelerntes einmal nützen soll «"1. Die - auch - »bildungsökonomischen« Grundlagen der Curriculum-Theorie, insbesondere auch die Fragwürdigkeit des Ansatzes zur Orientierung der Lernziele an "Situationen «, in denen strukturelle gesellschaftliche Bezüge allzu leicht auf, wenn auch konflikttheoretisch polarisierte, so doch subjektive Interaktionszusammenhänge reduziert werden, können und sollen von uns nicht analysiert werden ${ }^{22}$. Die notwendig fragmentarische Darstellung der Curriculum-Entwicklung soll hier nur soweit geführt werden, daß die Rechtsstruktur der Schule und insbesondere neue Ansätze zu deren Befestigung in ihrer Vermittlung mit den dadurch positiv bzw. negativ sanktionierten pädagogischen Konzeptionen verständlich werden. Die Lernzielorientierung der schulischen Praxis tendiert jedenfalls dazu, die Schulstruktur insgesamt dadurch zu verändern, daß der Bildungsprozeß »realistischer" wird und dadurch notwendigerweise auch politische Widersprüche reflektiert, die durch die bisherige Funktionsweise der Schule, insbesondere durch die Illusion der "Gleichheit " und "Einheit « der Schule für alle und unter der Form der Neutralität und Distanz zu den "konkreten Tagesfragen «, zu denen auch die konkreten sozialen Lebenssituationen der Schüler weitgehend gehörten, verdrängt waren. Insbesondere der Versuch, die »Selbstbestimmung « der Schüler nicht als von deren Praxis abgelöste staatliche »Erziehungsaufgabe « zu postulieren, sondern in einem Proze $\beta$ der Bestimmung und Realisierung von Lernzielen durch eine nicht nur formale "Beteiligung am Unterricht» sondern durch Involvierung

17 Vgl. dazu näher U. K. Preuß: Bildung und Herrschaft, Frankfurt/M. 1975, $74 \mathrm{ff}$.

I8 P. Bourdieu//. C. Passeron: Grundlagen einer Theorie der symbolischen Gewalt, Frankfurt/M. I 973 57.

19 Vgl. aus der umfangreichen Literatur nur G. Köhler/E. Reuter (Hg.): Was sollen Schüler lernen? Frankfurt/M. r973 und G. Köbler (Hg.): Wem soll die Schule nützen?, Frankfurt/M. 1974.

20 Vgl. nur das Interview mit Saul B. Robinsobn, in: Curriculumdiskussion, hg. von der Redaktion de Zeitschrift betrifft: erzıehung Wemheım/Basel 1974, 3 I ff., sowı die weiteren in diesem Buch enthaltenen Beiträge.

2i Der Hessısche Kultusmınıster: Rahmenrichtlinıen Sekundarstufe I, Gesellschaftslehre, Frankfurt/M. $1973,7 \mathrm{ff} ., 9$.

22 Vgl. dazu aber $U . K$. Preuß, a. a. O., insbes. $92 \mathrm{ff}$. 
ihrer Interessen zu erreichen, gefährdet das Verhältnis der »interesselosen « Trennung der Schüler - wie der Lehrer - von den auf unterschiedlichem Niveau jeweils zu rezipierenden, in Ausübung des staatlichen "Erziehungsrechts « formulierten und in der Schule quasi nur noch zu vollstreckenden staatlichen Bildungsinhalten. Die bisherige juristische Form der Bewältigung dieser Entwicklung zeichnet sich charakteristischerweise dadurch aus, daß alternative Modelle der Curriculumentwicklung umstandslos auf das bisherige Modell als Referenzebene der "Normalität» bezogen werden und die so als "anomal « diagnostizierten Abweichungen mit dem Verdikt der "Verfassungswidrigkeit « belegt werden ${ }^{23}$. Die Ansätze zu einer realistischeren schulischen Praxis, die insbesondere die reale Differenz der gesellschaftlichen Stellungen, für die die Schüler »fabriziert« werden, zu reflektieren suchen, werden an dem ideologischen Modell der sich selbst vollziehenden, von den gesellschaftlichen Interessen abgelösten schulischen »Einheit«, die die Qualifikation in "selbständigen « neutralen, für alle gleichen schulischen Zensuren etc. mißt, gemessen und, weil tendenziell nicht "selbständig « und abstrakt, als "einseitig", politisch und damit verfassungswidrig verworfen.

\section{3. "Neutralität« der Schule als staatliche Verwaltung}

Die Tendenz, die als »normal« und unproblematisch erlebte abgelöste, selbständige "Einheit« der Schule zu erhalten und eine konfliktorientierte Lernzielbestimmung als verfassungswidrig zu eliminieren, bedient sich in neuerer Zeit insbesondere einer Hypostasierung von "Toleranz « und »Neutralität « des Staates, die zugleich umstandslos auf die Schule als Staatsapparat ausgedehnt wird. Toleranz wird als fundamentaler Grundsatz für das gedeihliche Zusammenleben von Menschen unterschiedlicher Weltanschauung und Lebensauffassung bezeichnet, der in der Bundesrepublik seit dem 2. Weltkrieg als Ideal anerkannt sei ${ }^{24}$. Das Grundgesetz »huldige « diesem Prinzip vor allem im Grundrechtsteil, ohne dies allerdings ausdrücklich zu normieren. Der Staat als friedensstiftende Ordnungsmacht müsse Toleranz absichern und gewährleisten ${ }^{25}$. „Um das zu tun, muß er sich gegenüber den verschiedenen Anschauungen unparteiisch und neutral verhalten, er muß Zurückhaltung üben und darf sich insbesondere nicht mit einer Anschauung identifizieren ${ }^{26}$. „Der Staat, genauer die jeweilige Mehrheit, muß vielmehr bestrebt sein, ungeachtet ihres eigenen Standpunktes das staatliche Leben so auszugestalten, daß alle Gruppen oder zumindest fast [!] alle Gruppen den Staat auch als ihren Staat empfinden und mittragen können ${ }^{27}$. Die Schule muß sich in dem Rahmen halten, der durch den "Grundkonsens der Demokraten « als »Normalität « abgesteckt wird. Jede bestimmte, »einseitige « Festlegung der Schule ist nach dieser Konzeption unzulässig. Die eigentliche Gefahr für die Toleranz droht dabei gerade von denjenigen Gruppen und Meinungen, die den Grundkonsens der Demokraten nicht mittragen. Diese Gruppen sind daran zu identifizieren, daß sie auf einer bestimmten Meinung und deren

${ }_{23}$ Vgl. G. Püttner: Toleranz und Lehrpläne für Schulen, DOV 1974, 6g6ff., vgl. auch die Anfrage des Abgeordneten Dr. Kraske (CDU/CSU) betreffend die Lesebücher „drucksachen « 1m Bundestag ( 140. Sitzung) am Is. 1. 1975: "Würden Sie mir zustımmen, daß. Schullesebücher in ihrer Tendenz auch nicht Grundhaltungen konterkarieren sollten, - und zwar systematisch, wie es hrer geschehen ist -, die der Politik aller in diesem Hause vertretenen Parteien und der Regierung entsprechen . "(hier zitiert nach "Das Parlament 4, Nr. 6 v. 8. 2. 1975, 14).

24 Vgl. G. Püttner, a. a. O., 6,6 .

25 Vgl. G. Püttner, a. a. O., 657.

26 Vgl. G. Püttner, a. a. O., 657.

27 Vgl. G. Püttner, a. a. O., 657 . 
Richtigkeit insistieren und gegen andere Meinungen polemisieren, statt die eigene Meinung als relative zu begreifen, die anderen, ebenso legitimen gegenüber steht. "Die Anhänger neuerer Anschauungen, zum Beispiel des Neomarxismus, verstehen sich selbst nicht als Anhänger einer Weltanschauung neben anderen, sondern behaupten, wissenschaftlichen Erkenntnissen zu folgen, die allgemeine Anerkennung erheischen ${ }^{28}$. Solche Anschauungen müssen von der Schule ferngehalten werden, wobei das Berufsverbot allein nicht ausreicht. "Der Staat . . . kann extreme Gruppen, die Toleranz und ,Pluralismus` gänzlich beseitigen wollen, nicht dulden ${ }^{29}$.

Diese, in Rechtsform transformierte autoritäre Variante der Pluralismusdoktrin orientiert sich - unter Verkehrung bestimmter Ausnahmeregelungen des Grundgesetzes (Parteienverbote etc.) zur Regel der sogenannten streitbaren Demokratie auf kaum verhüllte Weise an dem Status quo einer bestehenden politischen und ökonomischen Machtstruktur und legitimiert ihren freiheitlichen pluralistischen Charakter allenfalls daran, daß dessen substanzhaft abgeschlossene Einheit mit integrativen Mechanismen der Konfliktregelung einerseits und der freien Auswahl der Machteliten (»Wechselspiel « von Regierung und Opposition) abgesichert wird. Sie legitimiert sich nicht - wie vorwiegend in anderen westeuropäischen Ländern durch die Institutionalisierung bestimmter, vor allem Gewaltfreiheit intendierender, formaler Verfahren gesellschaftlicher demokratischer Meinungs- und Willensbildungsprozesse, die ihre jeweilige Zuständlichkeit selbst transformieren können, sondern sie setzt einen historisch bestimmten "freibeitlichen "Zustand als Referenzebene für alle politischen Veränderungen des Prozesses selbst, der durch staatlich institutionalisierte Macht potentiell oder real auch der Verfügung durch demokratische Willensbildung des Volkes selbst entzogen wird. Das Bundesverfassungsgericht hat dies in dem KPD-Urteil (dem »heimlichen Grundgesetz « der BRD) als Grundsatz wie folgt proklamiert: "Diese freiheitliche demokratische Ordnung nimmt die bestehenden, historisch gewordenen staatlichen und gesellschaftlichen Verhältnisse und die Denk- und Verhaltensweisen der Menschen zunächst als gegeben hin ${ }^{30}$.

Dies ist der Hintergrund, vor dem sich die diversen »Toleranzgebote «, insbesondere auch für die Schule, darstellen und ein immer dichter werdendes Netz politischer Uniformisierung bilden, das so die als exzeptionell konzipierten und mit besonderen rechtlichen Verfahren verbundenen Repressionsmöglichkeiten immer mehr zur Regel macht. Systemkritische Ansätze werden als "anomal in einem faktischen und normativen Sinne stigmatisiert, über deren "Zulässigkeit" nach Gesichtspunkten polizeilicher Opportunität verfügt wird, einer Opportunität, die jedenfalls die Eliminierung von Systemkritik und Systemkritikern aus dem staatlich institutionalisierten Bereich verlangt. "Neutralität« des Staates sanktioniert die traditionelle harmonisierende Pädagogik und ihre Verfahren - vgl. dazu oben - und institutionalisiert damit einen Prozeß der »Neutralisierung " der Subjekte als »Toleranz" gegenüber allen Interessen und Situationen, mit denen die Subjekte sich konfrontiert sehen. "Ausgleich der Interessen « aller, »Güterabwägung " als internalisierte Praxis verlangen, daß die Schule die spezifischen Interessen von Subjekten als künftigen Lohnabhängigen ignoriert, weil sie eben die Schule "für alle « ist und die faktischen Unterschiede in der Gleichheit vor dem verselbständigten neutralen »Qualifikations«-Maßstab der Schule aufhebt.

Eine politische Pädagogik, die mit positiv besetzten Begriffen wie »Toleranz«, 
"Freiheit«, "Pluralismus « operiert, reproduziert unter dem Schein der Abstinenz des Staates von Manipulation die stets vorausgesetzte »Unmündigkeit « des Schülers, dessen Persönlichkeit noch "unvollständig " ist. Diese Pädagogik ist antithetisch fixiert auf die Motivation durch spezifische Interessen (insbesondere von Lohnabhängigen), die nicht in die »allgemeine Persönlichkeit «, wie sie durch die schulische Erziehung erst erworben wird, integriert sind. Die aus Schulabschlußfeiern kolportierte Redewendung von der "Entlassung ins Leben « hat ihren realen Sinn in diesem Verständnis des Erwerbs von »allgemeiner Persönlichkeit « in der Schule und ihrer "Anwendung i in den je besonderen Konflikten "des Lebens", in denen sich die "Entfaltung " der Selbstbestimmung durch die freiwillige Verwirklichung der in der Schule erlernten allgemeinen Verhaltensmuster in »eigener "Verantwortung vollziehen soll.

4. Das positivierte »Toleranzgebot «

Auch das in einigen Landesverfassungen explizit enthaltene Toleranzgebot versuchen die Protagonisten der »neutralen Schule« für die rechtliche Restriktion der Bildungsreform zu operationalisieren. Art. 56 Abs. 3 der hessischen Verfassung etwa lautet: »Grundsatz eines jeden Unterrichts muß die Duldsamkeit sein. Der Lehrer hat in jedem Fach auf die weltanschaulichen Empfindungen aller Schüler Rücksicht zu nehmen und die religiösen und weltanschaulichen Auffassungen sachlich darzulegen.« Dieses pädagogische Toleranzgebot ist ein Versuch der Verarbeitung der schulischen Entwicklung im Faschismus, der in erster Linie ein pädagogisches Verfahren fixieren will, nicht aber materiell bestimmte, auch politische Festlegungen ausschließen will. Das Toleranzgebot hat nur sekundär rechtlichen Charakter in dem Sinne, daß das pädagogische Prinzip der Beachtung unterschiedlicher Sozialisationsbedingungen und der dadurch entwickelten unterschiedlichen weltanschaulichen Orientierungen auch rechtlich verbindlich ist. So wenig es ein pädagogisches Gebot zur Toleranz in dem Sinne geben kann, daß es die "Beeinflussung « der Schüler in einem »bestimmten« Sinn verböte, so wenig kann es ein entsprechendes Rechtsgebot geben. Durch die unterschiedslose Subsumierung der gesamten Tätigkeit des Lehrers unter das Schema der verbindlichen Verwaltungsrechtsentscheidung wird unter dem Schein der Wahrung individueller Freiheit die freie Entwicklung diskursiver Lemprozesse restringiert, wenn Lehrer - wie auch Schüler ${ }^{31}$ - zu politischer Toleranz im Sinne von »Neutralität « verpflichtet werden. Die oben näher bezeichneten Tendenzen funktionieren die Pflicht zur Duldsamkeit und Rücksichtnahme auf andere weltanschauliche Positionen schließlich so weit um, daß schon die Äußerung einer eigenen kontroversen Position als unduldsam erscheint und durch Verhinderung der öffentlichen Kontroverse die Position selbst eliminiert wird. An dieser Verfassungsnorm läßt sich besonders deutlich demonstrieren, daß ein Verfahrensgebot dazu umfunktioniert wird, den in der Schule sich vollziehenden öffentlichen Lernprozeß in einem bestimmten Zustand von »Normalität« zu petrifizieren und das Toleranzgebot als Apperzeptionsfilter im Kopf des Schülers wie des Lehrers zu institutionalisieren. Bei näherem Hinsehen wird denn

31 Vgl. dazu VG Karlsruhe, Urteil yom 26. 10. 1972, abgedruckt in SPE II D II, 2I ff. Das Gerıcht überprüft eın von emer linken Schülergruppe verteiltes Flugblatt auf seıne politısche „Richtıgkeıt « und kommt zu dem Ergebnıs, daß die »allgemeıne Aussage des Flugblatts eıne „erhebliche Beeınträchtıgung « der - auch staarsbürgerlichen - Erzıehungsverantwortung* der Schule darstelle, weil ihr ein "simplifizıertes Vorverständnıs" von Gesetzgebung und "ungehemmte politısche Demagogıe" zugrundeliege. 
auch hier deutlich, daß die Protagonisten der »Toleranz " ganz explizit die Erziehung zu einer "positiven Einstellung “ postulieren ${ }^{32}$, deren Inhalt wiederum mit dem oben gekennzeichneten substanzialisierten, "Normalität « reproduzierenden Freiheitsbegriff identisch ist. Diese staatlich vorgegebene "Sachlichkeit « und "Toleranz" gegenüber dem Status quo der bestehenden politischen und gesellschaftlichen Verhältnisse hat der Lehrer als Vorbild ${ }^{33}$ seinen Schülern vorzuleben ebenso wie in der Bundeswehr der Vorgesetzte seinen Soldaten ${ }^{34}$. Freiheit als Verfahren der Entwicklung und Selbstdefinition von Interessen gilt nur noch für die nicht institutionalisierten "gesellschaftlichen « Bereiche - und auch dort nur unter Opportunitätsgesichtspunkten so lange, wie die Verteidigungsbereitschaft der »streitbaren Demokratie « nicht gefährdet ist. Innerhalb des als staatlich aggregierten, öffentlichrechtlich geregelten Bereichs ist der Begriff der Freiheit staatlich substanzialisiert und an den Erfordernissen der Reproduktion des Status quo orientiert.

$\mathrm{Daß}$ diese Pflicht zu "Neutralität « und »Toleranz« nicht nur dem Staatsapparat die illusionäre Pflicht zur politischen Abstinenz auferlegt, manifestiert sich auch darin, daß diese "Neutralität« als "Erziehungsaufgabe« explizit gegen den Schüler gewendet wird, der in der Schule Auffassungen verbreitet, die mit dem substanzialisierten Freiheitsverständnis unvereinbar sind 35 . Und daß just dies auf eine staatliche Kontrolle politischer Meinungsbildung von Schülern auf ihre "Richtigkeit « hinausläuft, und zwar diesmal tatsächlich mit potentiell rechtsverbindlichen Sanktionen, wird nicht dadurch erträglicher, daß diese Kontrolle sich auf bestimmte öffentlichrechtlich "ein- « und von der "Gesellschaft « "ausgeschlossene « Bereiche beschränkt ${ }^{36}$. Die Verteidiger der "Toleranz« im Schulwesen identifizieren also nicht nur eine ganz bestimmte Konzeption von Pädagogik mit der Verfassung, die mit den wenigen einschlägigen Normen nur eine rechtliche Intervention in den schulischen Bildungsprozeß vorgenommen hat, ohne dessen Prozeßcharakter im Sinne einer bestimmten Pädagogik zu fixieren und als Gegenstand demokratischer, öffentlicher Transformation zu entziehen. Sie müssen darüber hinaus konsequenterweise die Internalisierung der so substanzialisierten Bildungsziele durch die Schüler selbst notfalls mit Zwangsgewalt durchsetzen ${ }^{37}$, während etwa gerade der situative Ansatz der Rahmenrichtlinien durchaus eine formale Freiheit im Sinne von Selbstdefinition verschiedener "Lösungsmöglichkeiten " ausdrücklich voraussetzt ${ }^{38}$. Auch daran wird noch einmal deutlich, daß die "Freiheitlichkeit « der Pluralisten in der Schule wie übrigens in der Wissenschaft insgesamt - sich begrifflich in nichts anderem konstituiert als einer Art von »bildungspolitischem «bzw. »wissenschaftstheoretischem Kontrakt « (man könnte auch von "großer Koalition « oder »Solidarität aller Demokraten « sprechen), der das »Wechselspiel« der politischen Parteien wie der wissenschaftlichen Hypothesen (»kritischer Rationalismus«) auf der Basis der Verteidigung des Status quo in der antithetischen Fixierung auf politische wie wissenschaftliche "Systemveränderer « institutionalisiert. Die Funktionsweise dieses antithetischen Pluralismus stilisiert einen als Normalität verstandenen Status quo begrifflich zur Referenzebene und vereinnahmt dadurch die Differenz anderer Ansätze (politischer wie wissenschaftlicher) als »Anomalität«. (Symptomatisch dafür ist

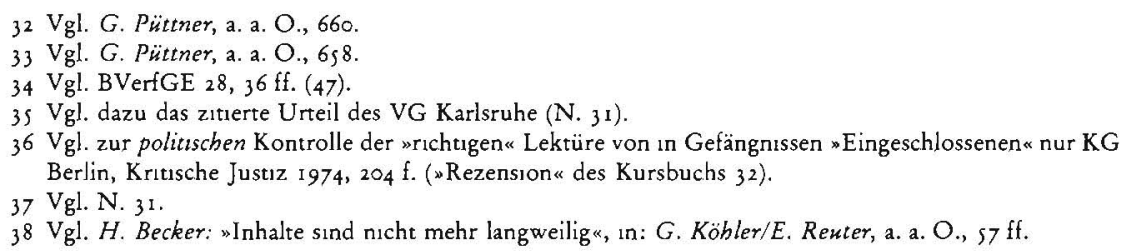


die Bezeichnung der wissenschaftlichen Begründung der Rahmenrichtlinien als »wissenschaftlich « ${ }^{39}$ ).

\section{Zwischenergebnis}

Die Tendenz zur Verrechtlichung der besonderen Gewaltverhältnisse der Schüler (wie der Lehrer) hat - wie gezeigt - nicht nur zu keiner Erweiterung von grundrechtlicher Freiheit in der Schule geführt, sondern im Gegenteil zu deren Einschränkung. Die Verrechtlichung der Aktionsräume der Bildungsreform hat objektiv die Funktion, die Aktionsräume vor allem sozialdemokratischer Kultusbürokraten, die zur Revision von Curricula durch "realistischere" situative Lernzielorientierung bereit waren, zu restringieren. Die Verrechtlichung der der Gewaltunterwerfung des Schülers entsprechenden Aktionsfreiheit des Staatsapparates soll insgesamt die Kontrolle der Bildungsinhalte durch die Gerichte, deren »Neutralität « zuverlässiger zu sein scheint als die der augenscheinlich für »Politisierung « mindestens partiell anfälligen Kultusbürokratien, ermöglichen und so den Gerichten die Wahrung der "Legitimität« der Bildungspolitik anvertrauen. Diese Einschätzung erscheint wegen der Verwandschaft der traditionellen Schul- und Rechtsideologien (die Güterabwägung ist der Besinnungsaufsatz des Richters, wie der Besinnungsaufsatz die Güterabwägung des Schülers ist!) durchaus realistisch ${ }^{\circ}$. An die Stelle der traditionellen Reproduktionsform der Einheit der Staatsperson tritt so eine andere »Einheit" stiftende Instanz, das Gericht, das als Schlichtungsinstanz zwischen den "pluralistischen « Positionen bzw. zwischen Staat (als politischer Verwaltung und politischer Gesetzgebung) und den Individuen Einheit repräsentieren muß, weil die Einheit der Staatsperson durch den "Pluralismus « innerhalb der Gesetzgebung wie der Verwaltung mindestens partiell aufgebrochen worden ist und eine neue "einheitliche ", »neutrale« Darstellungsebene braucht, die im Gericht rekonstruiert wird.

\section{Elternrecht}

Auch die in neuerer Zeit wieder zu registrierende Expandierung des "pädagogischen Elternrechts « ist vor allem unter dem Ziel der Ausdehnung der Gerichtskontrolle betrieben worden ${ }^{41}$. Das extensive Verständnis des Elternrechts soll den wörtlich so verstandenen "Bereich “ der "Persönlichkeitsbildung " im Gegensatz zur "Wissensvermittlung « den Eltern zur ausschließlichen Einwirkung vorbehalten ${ }^{42}$. In Art. 6 Abs. 2 Satz I GG heißt es: "Pflege und Erziehung der Kinder sind das natürliche Recht der Eltern und die zuvörderst ihnen obliegende Pflicht«. Die Auslegung dieser Norm war zur Zeit der Entstehung des Grundgesetzes kaum problematisch ${ }^{43}$. Als Grundrecht konnte sie nur die individuellen Erziehungsmöglichkeiten der

39 Vgl. G. Püttner, a. a. O., 559.

40 Die juristische Ideologie als die »klassische der Bourgeoısıe (F. Engels: Juristensozıalismus, in: MEW $2 \mathrm{I}, 49 \mathrm{I}$ ff. [492]) reproduzıert sıch innerhalb der Darstellungsbedingungen der Pädagogik ınsbesondere in den traditionellen Erziehungszielen, in denen sıch daher auch das Gerıcht »wiedererkennen " kann.

$4 \mathrm{I}$ Vgl. insbesondere zum "Spannungsverhältnis « $z w_{1 s}$ schen Elternrecht und "staatlicher Sexualerzıehung * H. U. Evers, a. a. O. (N. S); H. Scholzen: Staatliche Sexualerziehung, RdJB 1974, $216 \mathrm{ff}$.; R. Stober: Sexualkunde in den Schulen?, DOV $1973,556 \mathrm{ff}$.

42 Vgl. auch die oben (N. 4I) zitierten Autoren zu einer *vermittelnden* Meinung, die einen wdritten Bereıch $\approx$ zu erkennen vermeınt, in dem das durch die Gerıchte vermittels »Güterabwägung « jeweils ım Einzelfall zu bestimmende »überwıegende Interesse « zu Gunsten des Staates oder zu Gunsten der Eltern Ausschlag geben soll.

43 Vgl. I. Richter: Bildungsverfassungsrecht, Stuttgart 1973 , $55 \mathrm{f}$. 
Eltern vor staatlichen Eingriffen zu schützen intendieren. Der Staat darf den Eltern nicht die Möglichkeit nehmen, die Kinder in der Familie zu erziehen. Daß diese Möglichkeit durch den schulischen Unterricht nicht tangiert wird, dieser also nicht als Eingriff in das Recht der Eltern aufgefaßt werden kann, war daher zunächst kaum streitig, zumal Art. 7 Abs. I GG (staatliche Schulaufsicht) gleichrangig neben dem Elternrecht steht. Ebensowenig wie die verschiedensten außerfamiliären sozialen Kontakte, denen das Kind ausgesetzt ist, nimmt der Unterricht den Eltern auch bei Bestehen der allgemeinen Schulpflicht die Möglichkeit, auf ihr Kind einzuwirken. Die faktischen schulischen Einflüsse, die durchaus das Erziehungskonzept der Eltern konterkarieren mögen, müssen außerhalb der rechtlichen Betrachtungsweise bleiben. Die gegenteilige Auffassung müßte übrigens konsequenterweise den Eltern ein Verbot auferlegen, ihrerseits durch eigene abweichende Meinungen die "Einwirkungen « der Schule auf den ihr vorbehaltenen Bereich der "Wissensvermittlung « zu behindern. Das Elternrecht kann nicht die Kompetenz enthalten, rechtlich verbindlich gegenüber jedermann die Grundlinien der Erziehung zu regeln und festzulegen, weil dies dem Charakter der Erziehung und Bildung als Prozeß zuwiderlaufen würde. Ein derart extensiv interpretiertes Elternrecht würde - ernst genommen jeglichen staatlichen Unterricht unmöglich machen können. Die propagierte Erweiterung führt denn auch nur scheinbar zu einer Erweiterung der materiellen Bestimmungs- und Mitbestimmungsrechte der Eltern. Nach den oben gemachten Ausführungen liegt es auf der Hand, daß die Eltern als Individuen auch nur das einfordern können, was die Gerichte ihnen durch "Güterabwägung «44 als "normal « zuteilen. Die Abtrennung eines Erziehungsbereiches »Persönlichkeitsbildung" von einem Erziehungsbereich "Wissensvermittlung" ist natürlich illusionär. Diese Konstruktion hat nur die Funktion, das Klagerecht der Eltern als Verfabrensrecht zu legitimieren, weil das, was aufgrund einer Klage der Eltern tatsächlich, weil normal, durch Güterabwägung zugeteilt wird, dem Kläger nach der juristischen Darstellungsweise als eigenes Recht zustehen muß. Ein "anomaler " Gebrauch des Elternrechts würde, als "Gut « gewogen auf der Gerichtswaage, deren Gewichte auf "Normalität« geeicht sind, gegenüber dem jeweiligen "Gut« des Staates, zum Beispiel dem "staatlichen [!] Interesse an Bürgern, die der Sexualität ein vertieftes Verständnis entgegenbringen « 45 etc. etc., als zu leicht befunden werden.

\section{Zur "pädagogischen Freibeit des Lebrers}

Auf den engen Zusammenhang der politischen und pädagogischen Disziplinierung von Schülern und Lehrern ist in den bisherigen Ausführungen bereits hingewiesen worden. Die "pädagogische Freiheit « des Lehrers ist allerdings von der formalen rechtlichen Transformation des besonderen Gewaltverhältnisses fast völlig unberührt geblieben. Daß der Lehrer, von der Bindung an Gesetze, Lehrpläne, Erlasse usw. abgesehen, auch im übrigen einem in keiner Weise begrenzten Weisungsrecht der Schulaufsichtsbehörden unterliegt, wird trotz der auch in der rechtswissenschaftlichen Literatur laut gewordenen Zweife ${ }^{46}$ durchweg in den einschlägigen Landesgesetzen vorausgesetzt. Selbst dort aber, wo die "pädagogische Freiheit« ausdrücklich gesetzlich anerkannt ist, wie in Hessen, hat das Recht eher den Charakter des Lichtenbergschen Messers, dem bekanntlich weder Heft noch Klinge

44 Vgl. H. U. Evers, a. a. O., OVG Hamburg, a. a. O., I I ,

45 OVG Berlin, a. a. O., 554 .

${ }_{4} 6$ Vgl. nur W. Perschel: Die Lehrfreihert des Lehrers, DOV 1970, 34 ff. m.w.N. 
eigen sind, denn die pädagogische Freiheit besteht nur in dem Rahmen, den Lehrpläne und das rechtlich verbindliche, zugleich aber rechtlich voraussetzungslose Weisungsrecht der Schulaufsichtsbehörde dem Lehrer faktisch überlassen, sie ist aber rechtlich nicht größer als die eines jeden anderen Beamten auch. Symptomatisch ist eine in der "führenden « Schulrechtskunde ${ }^{47}$ an die Schulaufsichtsbehörden adressierte Mahnung, bei der Ausübung des Weisungsrechts die Grenzen des "Takts" zu beachten. Die notwendigerweise bestehende faktische Freiheit des Lehrers, der - anders als der typische Verwaltungsbeamte - nicht in einem mindestens potentiell auf Einheit durch Hierarchie angelegten rechtlich zernierten Verfahren der rechtsverbindlichen Entscheidungsbildung, sondern in einen pädagogischen Proze $\beta^{4^{8}}$ involviert ist, wird in der rechtswissenschaftlichen und pädagogischen Literatur teilweise allerdings auch als Recht auf pädagogische Freiheit eingefordert $^{49}$. Die Rechtsformen, in denen diese Forderung erhoben wird, scheinen uns kaum ihrem Gegenstand angemessen zu sein: Es kann nicht darum gehen, einerseits dem Recht des Staates und der Eltern noch ein weiteres Recht »am Kinde«, eben das Recht des Lehrers, hinzuzufügen oder andererseits ein abstraktes Individualrecht des Lehrers als »kleine Wissenschaftsfreiheit « dem traditionellen Verständnis des "Grundrechts des Hochschullehrers « nachzubilden ${ }^{\circ}$, ein Recht, das als abstraktes Individualrecht aus dem kollektiven Zusammenhang der Schule herausgelöst würde und dann durch begriffslose Abwägung gegen die anderen Rechte doch wieder auf das Maß der Normalität des Bestehenden reduziert würde. Eine alternative Rechtskonzeption müßte vielmehr gerade die Freibeit der Schule als Prozeß erhalten, in den das Recht interveniert, ohne aber den pädagogischen Prozeß in der langsamen und gleichförmigen Bewegung der Normalität substanziell zu fixieren und durch Güterabwägung stets aufs Neue zu reproduzieren. Diese Rechte müßten vielmehr als "Reflexrechte « konzipiert werden, deren Verhältnis nicht durch Güterabwägung sondern durch die Entwicklungsbedingungen des Prozesses Schule selbst miteinander vermittelt $\operatorname{sind}^{51}$. Die praktischen Konsequenzen einer solchen hier nur angedeuteten Konzeption sollen nur kurz zusammengefaßt werden: Die pädagogische Freiheit des Lehrers schließt die staatliche Fixierung von bestimmten Bildungszielen keineswegs aus. Eine Pflicht zur »Neutralität der Lehrpläne « besteht (weil unerfüllbar) nicht, die Fixierung von Bildungszielen muß vielmehr dem bildungspolitischen Prozeß, das heißt letztlich: der jeweiligen politischen Mehrheit überlassen bleiben. Insoweit besteht Raum für unterschiedliche Formen der Organisierung und Aggregierung von - auch - politischen und wissenschaftlichen Kommunikationsprozes-

47 Vgl. H. Heckel/P Serpp: Schulrechtskunde, 4. Aufl., Neuwed-Berlin 1969, 2 is.

$4^{8}$ Die Reduktıon des pädagogıschen we anderer Prozesse auf eınen hierarchisch strukturıerten Entsche1dungsstrang wird oft und gern mit der Notwendigkeit der über die "Verantwortlichkett « des Minsters zu vermittelnden parlamentarischen Kontrolle legitimiert, vgl. dazu $U$. Kollatz: Freiheıt des Lehrers vom Grundgesetz? - Erwiderung zum Aufsatz Perschel in DOV 1970, 34, DOV 1970, 594, 595. Diese Argumentatıon bedürfte eıner besonderen, hier nicht zu leıstenden Analyse. Hier seı nur angemerkt, daß die parlamentarısche Kontrolle andererseıts natürlich durch die "Gewaltentrennung " begrenzt wird, die eıner rechtsverbundlichen Einzelweısung an den Minıster entgegensteht. Schon dies zeıgt, daß das oben zitıerte Argumentationsmuster Ursache und Wirkung vertauscht: die parlamentarısche Kontrolle dient nur der Legitimation der Hierarchisierung, nicht umgekehrt dient die Hierarchisierung der parlamentarischen Kontrolle. Mit der gleıchen Argumentation könnte übrıgens auch die Wissenschaftsfreiheıt an den Universitäten eliminert werden, zumal deren besondere Legitimation durch wfreie Wahl der Ausbildungsstätte - Im Gegensatz zur Erfüllung der Schulpflicht in einer bestımmten, nämlich der Schule, in deren Schulbezırk sıch der Wohnsıtz des Schülers befinder - (vgl. dazu U. Kollatz, a. a. O., 554) infolge des numerus clausus in zunehmendem Maße entkräftet wird. Wer schützt also die Studenten vor der Lehrfreihest?

49 Vgl. W Perschel, a. a. O.

so Vgl. einerseits E. Reuter, in: G. Köhler/E. Reuter (N. 19), 30 und andererseits W Perschel, a. a. O.

sı Vgl. zum Prozeßcharakter der politıschen Grundrechte demnächst $H$. Ridder, ın: Bad Wildunger Beiträge zur Gemeinschaftskunde, Bd. s (hg. J. Mück), Köln-Opladen r975. 
sen. Aber diese Festlegung darf den Prozeßcharakter der Reproduktion gesellschaftlichen Wissens in der Schule nicht dadurch aufheben, daß durch die Verbindlicherklärung bestimmter politischer, wissenschaftlicher und weltanschaulicher Positionen die Entwicklung "abweichender « Meinungs- und Bildungsprozesse der Schüler wie der Lehrer juristisch negativ sanktioniert werden. Die Vorgabe von Bildungszielen und Bildungsinhalten durch "Lehrpläne", Curricula etc. darf die kritische Reflexion dieser Inhalte selbst nicht inhibieren. Die in der Schule ungeachtet ihrer Rechtsform als staatliche unselbständige Anstalt sich entwickelnde Dialektik des pädagogischen Prozesses darf in ihrer Struktur durch staatlich kompetenzielle Intervention (in Form von "Lehrplänen« etc.) fixiert werden, aber die inhaltliche Entwicklung der vorgegebenen Struktur muß dem so organisierten Prozeß in der Weise grundrechtlich zur nichtstaatlich fixierten Selbstdefinition überlassen werden, daß alle politischen Meinungen (Art. s Abs. I GG), soweit sie auch außerhalb der Schule inhaltlich nicht gegen die "allgemeinen Gesetze« etc. (Art. s Abs. 2 GG) verstoßen würden, sowie alle wissenschaftlichen Konzeptionen (Art. s Abs. 3 GG) auch innerhalb des in der Schule aggregierten Teils des öffentlichen Meinungs- und Willensbildungsprozesses zulässig sind.

"Toleranz« als pädagogische (und juristische) Verfahrensregel könnte so erst ihren Sinn erhalten. 